\title{
Anabases
}

ANABASES Traditions et réceptions de l'Antiquité

1 | 2005

Varia

\section{Leandro POLVERINI (éd.), Aspetti della storiografia di}

\section{Ettore Pais}

\section{Sarah Rey}

\section{OpenEdition}

\section{Journals}

Édition électronique

URL : http://journals.openedition.org/anabases/1512

DOI : 10.4000/anabases. 1512

ISSN : 2256-9421

\section{Éditeur}

E.R.A.S.M.E.

\section{Édition imprimée}

Date de publication : 1 mars 2005

Pagination : 306-307

ISSN : 1774-4296

\section{Référence électronique}

Sarah Rey, «Leandro polverin (éd.), Aspetti della storiografia di Ettore Pais », Anabases [En ligne], 1 | 2005, mis en ligne le 01 octobre 2011, consulté le 22 septembre 2020. URL : http://

journals.openedition.org/anabases/1512 ; DOI : https://doi.org/10.4000/anabases.1512

Ce document a été généré automatiquement le 22 septembre 2020.

(c) Anabases 


\title{
Leandro POLVERINI (éd.), Aspetti della storiografia di Ettore Pais
}

\author{
Sarah Rey
}

\section{RÉFÉRENCE}

Leandro POLVERINI (éd.), Aspetti della storiografia di Ettore Pais, Naples, Edizioni

Scientifiche Italiane, $2002,352 \mathrm{p}$.

28,50€ / ISBN 88-495-0535-3

1 En France, Ettore Pais demeure méconnu. Pour beaucoup, Pais n'est que l'historien hypercritique des origines de Rome. Certains ont peut-être gardé en mémoire le volume de l'Histoire générale de Gustave Glotz qui lui a été confié. D’autres savent son allégeance au fascisme. Et c'est souvent à peu près tout. Ettore Pais (1856-1939) mérite pourtant plus de curiosité : c'est un homme singulier, «voyant» (p.9), auteur d'une œuvre majeure. Cette œuvre a longtemps souffert de deux jugements sévères portés sur elle: celui de Benedetto Croce, qui a dit n'avoir que « dégoût » pour l'historien dévôt de Mussolini; et celui de Piero Treves dans Lo studio dell'antichità classica nell' Ottocento (1962), vraie bible sans équivalent sur l'histoire des études classiques italiennes au XIX ${ }^{\mathrm{e}}$ siècle. Mais cette bible est par trop hostile à Pais, à qui Treves n'a pas non plus pardonné son engagement du côté du fascisme.

2 Sans laisser indifférent, le parcours politique d'Ettore Pais ne fait plus aujourd'hui autant ombrage à la compréhension de ses travaux. Et les sentences sans retour de Croce et Treves peuvent être mises un temps de côté pour qu'apparaisse dans toute sa complexité l'itinéraire scientifique de Pais.

3 Nés des quatrièmes Rencontres de l'Université de Pérouse consacrées au monde des antiquisants, les Aspetti della storiografia di Ettore Pais jettent les bases, solides, de la biographie intellectuelle à laquelle le savant italien a justement droit. Ces Aspetti apportent beaucoup au lecteur désireux de connaître l'histoire de la science italienne de l'Antiquité, de la fin du XIX ${ }^{\mathrm{e}}$ siècle au fascisme. L'ouvrage est construit en deux 
temps, et douze contributions réparties symétriquement. La première partie (six contributions) se veut avant tout biographique, étudiant les rapports entretenus par le savant avec l'Allemagne et les pays anglophones, analysant de même son nationalisme, si discuté et si longtemps stigmatisé, nationalisme qui le pousse à prendre rapidement parti pour un rattachement à l'Italie des terres irrédentes. Deux articles donnent en outre à voir le Pais des années troubles, sénateur à vie (1922-1939), qui envoie à l'occasion d'obséquieuses lettres au Duce. La seconde partie des Aspetti, soit six autres contributions, plonge dans les travaux de l'antiquisant et retrouve les grands traits de Pais épigraphiste (parmi les meilleurs), numismate, spécialiste de Strabon, historien de la Sardaigne romaine et de la Sicile antique. L'infructueuse tentative de réorganisation de l'Officina dei papiri d'Herculanum par Pais au moment où il dirige le Musée national de Naples est, elle aussi, décrite.

4 Ce recueil est composé d'études rigoureuses et vivantes, où l'on apprend par exemple comment, au cours de son enfance en Sardaigne, le petit Pais, à peine âgé de six ans, aurait rencontré le grand Garibaldi, qui l'aurait pris sur ses genoux ; comment - aussi Ettore Pais, occupé à rassembler les inscriptions de l'ancienne Cisalpine pour le Corpus Inscriptionum Latinorum., est pris pour un espion à la frontière franco-italienne; comment il accepte pour son malheur la charge de directeur du Musée national de Naples ignorant les avertissements de Theodor Mommsen sur la camorra napolitaine ; comment l'antiquisant songe un jour à ériger à Rome un double de la colonne trajane en l'honneur des héros du Risorgimento ; comment il n'y aurait jamais eu de chaire d'italien à l'Université de San Francisco sans Pais... Au-delà de l'anecdotique, ce même recueil révèle un Pais souvent inattendu, féministe, animé d'idées sociales, prêt à condamner l'assassinat de Matteoti et refusant de signer en 1925 le manifeste Gentile, manifeste des intellectuels fascistes. Dans ces Aspetti, ceux qui s'attachent le plus à faire découvrir un Pais déconcertant, humain, pas immédiatement converti au fascisme sont naturellement des chercheurs de l'Université de Sassari, venus réhabiliter avec mesure une gloire sarde. Mais ici, cependant, rien n'est caché non plus des ambiguïtés d'Ettore Pais dont les convictions nationalistes se radicalisent à partir de 1911, à l'heure de la conquête libyenne. C'est Pais qui applaudit un discours impérialiste du Duce, Roma antica sul mare (1926), dont il est peut-être lui-même l'auteur, et c'est Pais qui imagine au lendemain de la victoire italienne en Éthiopie un triomphe à l'antique pour Mussolini.

5 Mettant à jour la multiplicité du personnage Pais, le volume aide en outre à envisager les paradoxes de la réception réservée au fil des années à ses travaux. Passé par l'école de Mommsen en 1881-1883, Pais est étonnamment peu lu en Allemagne. A l'inverse, les Anglo-saxons, restés des années durant perplexes devant le travail de Pais, porteur « de beaucoup de profit, mais pas du moindre petit plaisir» (p. 44), seraient de nos jours parmi les lecteurs fréquents de la Storia di Roma. En dehors des espaces germanique et anglophone, on peut regretter que l'écho reçu en France par les livres d'Ettore Pais ne fasse l'objet que de quelques remarques concises dans le bilan conclusif...

$6 \quad$ En fin de lecture des Aspects de l'historiographie d'Ettore Pais émerge une figure contrastée et versatile, dont la sage vie d'antiquisant dit tout de même beaucoup de l'histoire culturelle de l'Italie risorgimentale puis mussolinienne. Les Aspetti, tout en faisant apparaître les évolutions et les contradictions de la vie du savant, dressent de Pais un portrait fragmenté qui ne paraît jamais disparate. 
7 L. Polverini, «Introduzione ». Prima Parte. Per la biografia intellettuale e politica di Ettore Pais: A. Marcone, "Pais e la Germania »; R. T. Ridley, «Ettore Pais and the English-speaking world »; M. Cagnetta, « Pais e il nazionalismo »; G. Bandelli, « Pais e il confine orientale d'Italia »; P. Ruggeri, « Ettore Pais sena-tore del Regno »; R. Visser, "The Correspondence of Ettore Pais in the "Segreteria particolare del Duce, Carteggio ordinario"». II Parte. Ettore Pais nella storiografia italiana sul mondo antico: M. Buonocore, «L'attività epigrafica"; N. Parise, "L'interesse di Ettore Pais per la numismatica antica »; M. Capasso, «Ettore Pais e l'officina dei papiri »; A. M. Biraschi, «Pais e Strabone»; A. Mastino, «Ettore Pais e la Sardegna romana »; G. Salmeri, « Ettore Pais e la Sicilia antica ». F. Càssola, « Bilancio conclusivo ».

\section{AUTEURS}

\section{SARAH REY}

Université de Toulouse-Le Mirail 